\title{
On the use of Generalized Rodrigues Parameters for Representing Rigid Body Rotations
}

\author{
Gabriele Giorgi
}

Received: 30.10.2018 / Accepted: 21.05.2019

\begin{abstract}
This work complements the body of studies on the use of generalized Rodrigues parameters (GRPs) for describing the orientation of a rigid body. A simple decision logic is designed for handling the GRPs so that singular configurations are avoided, while enforcing the one-to-one mapping between the set of orientation parameters and the unique rotation matrix.
\end{abstract}

Keywords Rodrigues $\cdot$ quaternion $\cdot$ rotation · parametrization

\section{Introduction}

The instantaneous orientation of a rigid body in space is unambiguously described by a $3 \times 3$ orthonormal matrix with positive determinant and three degrees of freedom, hereafter denoted as $\mathbf{R} \in \mathbb{S O}(3)$, where $\mathbb{S O}(3)$ identifies the special orthogonal group of order $3[1,2]$. Among the available sets of attitude parameters used to represent the rotation matrix $\mathbf{R}$, the four-element quaternion of rotation [3] is widely employed, mainly due to the absence of singularities in both the direct (quaternion to rotation matrix) and inverse (rotation matrix to quaternion) transformations. Furthermore, the rotational kinematic equations can be expressed as linear functions of the quaternion. However, a unit norm constraint must be enforced, thus introducing nonlinearities in any control function using quaternions. To avoid the constraint, a number of alternative representations were devised based on different combinations between the elements of a quaternion. One of these parametrizations is the Rodrigues vector representation [4], also known as the Gibbs vector [5],

G. Giorgi

Institute of Communications and Navigation, German Aerospace Center (DLR)

Oberpfaffenhofen-Wessling, Germany

Tel.: +49-8153-28-2846

E-mail: gabriele.giorgi@dlr.de 
in which a three-element vector is obtained by forming the ratio between the vectorial $(\mathbf{q})$ and the scalar $\left(q_{0}\right)$ components of a quaternion:

$$
\mathbf{r}=\frac{\mathbf{q}}{q_{0}}
$$

This representation is singular for rotations of magnitude $\phi=(2 k+1) \pi$, with $k \in \mathbb{Z}$, about any axis $\mathbf{n}$. A modification of the Rodrigues vector representation was introduced in [6], and later analyzed in [7] and [8]:

$$
\mathbf{r}^{\prime}=\frac{\mathbf{q}}{q_{0}+1}
$$

This modification "pushes" the first singularity - if starting from the nullrotation - from $\pi$ to $2 \pi$, thus enabling a wider operation interval devoid of numerically unstable configurations. With this modification the singularities are located at rotations of magnitude $\phi=2(2 k+1) \pi$.

Following from the two-to-one mapping from quaternion to rotation $\mathbf{R}\left(q_{0}, \mathbf{q}\right)=$ $\mathbf{R}\left(-q_{0},-\mathbf{q}\right)$, the same orientation can be represented by either $\mathbf{r}^{\prime}$ or a so-called shadow set of parameters

$$
\mathbf{r}_{s}^{\prime}=\frac{\mathbf{q}}{q_{0}-1}
$$

This second set becomes singular for any rotation of magnitude $\phi=4 k \pi$. Combining the use of direct and shadow modified Rodrigues parameters enables describing any rotation unambiguously. The parameter set with the smaller norm is used to switch between between $\mathbf{r}^{\prime}$ and $\mathbf{r}_{\mathbf{s}}{ }^{\prime}$ [7]: this guarantees a bijective mapping between rotation and attitude parameters.

More recently, a generalization of the modified Rodrigues parameters was introduced in [9] and [10], in which a three-element vector is formed as

$$
\mathbf{p}=\frac{\mathbf{q}}{q_{0}+a}
$$

The elements of this set are known as generalized Rodrigues parameters (GRPs) or symmetric stereographic orientation parameters (SSOPs). An analysis and graphical interpretation of the stereographic projection that generates the GRPs is given in [9]. The scalar $a(|a| \leq 1)$ in (4) can place the singularity at any arbitrary rotation between 0 and $2 \pi$. However, for values of $a \notin\{-1,0,1\}$ this generalization introduces ambiguities in the mapping from the parameter set to the orientation matrix: the same set of GRPs generates two different rotations. Moreover, when inverting the transformation, the same rotation matrix maps to two different sets of GRPs. To resolve this duality, it was proposed in [10] - limited to the direct mapping - to employ one set of GRPs if the actual value of the quaternion scalar component $q_{0}$ exceeds $a$, or their shadow counterpart - obtained by switching the sign of $a$-if it does not. However, this demands an a-priori knowledge of the actual body orientation, which limits the operational use of GRPs.

It is shown in this work how to employ the GRPs without a-priori knowledge of the actual orientation, while still avoiding singularities and guaranteeing one-to-one transformations for both direct and inverse mapping. 


\section{Attitude parameterization with GRPs}

Equation (4) defines the relationship between the GRPs and the quaternion. This expression can be manipulated to solve for the quaternion as

$$
\begin{aligned}
& q_{0}=\frac{-a \mathbf{p}^{\mathrm{T}} \mathbf{p} \pm \sqrt{\left(1-a^{2}\right) \mathbf{p}^{\mathrm{T}} \mathbf{p}+1}}{\mathbf{p}^{\mathrm{T}} \mathbf{p}+1}=\xi-a \\
& \mathbf{q}=\xi \mathbf{p}
\end{aligned}
$$

where, for ease of notation, the scalar

$$
\xi=\frac{a \pm \sqrt{\left(1-a^{2}\right) \mathbf{p}^{\mathrm{T}} \mathbf{p}+1}}{\mathbf{p}^{\mathrm{T}} \mathbf{p}+1}
$$

has been introduced. Note that this expression explains the constraint $|a| \leq 1$, since values of $a$ outside of this interval may generate imaginary roots.

2.1 Direct mapping: quaternions to GRPs

Substitution of expressions (5) into the quaternion parameterization $\mathbf{R}\left(q_{0}, \mathbf{q}\right)=$ $\left(q_{0}^{2}-\mathbf{q}^{\mathrm{T}} \mathbf{q}\right) \mathbf{I}_{3}+2 \mathbf{q} \mathbf{q}^{\mathrm{T}}-2 q_{0} \boldsymbol{\Omega}_{\mathbf{q}}$ yields

$$
\mathbf{R}(\mathbf{p})=\mathbf{I}_{3}+2 \xi^{2}\left(\frac{a-\xi}{\xi} \Omega_{\mathbf{p}}+\boldsymbol{\Omega}_{\mathbf{p}}^{2}\right)
$$

with $\mathbf{I}_{3}$ the $3 \times 3$ identity matrix and $\Omega_{\mathbf{x}}$ the skew-symmetric matrix obtained from the three elements of vector $\mathbf{x}$ as

$$
\boldsymbol{\Omega}_{\mathbf{x}}=\left[\begin{array}{ccc}
0 & -x_{3} & x_{2} \\
x_{3} & 0 & -x_{1} \\
-x_{2} & x_{1} & 0
\end{array}\right]
$$

Expression (7) defines the attitude parametrization in terms of GRPs (see Appendix A.1 for a formal proof).

The relationship between GRPs and the corresponding Euler representation (rotation axis $\mathbf{n}$ and angle $\phi$ ) is

$$
\mathbf{p}=\frac{\mathbf{n} \sin \frac{\phi}{2}}{\cos \frac{\phi}{2}+a}
$$

Note that the inverse of the rotation matrix is obtained by inverting the sign of the GRPs:

$$
\mathbf{R}^{-1}(\mathbf{p})=\mathbf{R}(-\mathbf{p})
$$

This is easily demonstrated by inspection of expression (7): the transpose of matrix $\mathbf{R}(\mathbf{p})$ is obtained by inverting the sign of the non-symmetric component $\boldsymbol{\Omega}_{\mathbf{p}}$. 
Each pairing $(\mathbf{p}, a)$ produces two different matrices, hereafter denoted with $\mathbf{R}^{+}(\mathbf{p})$ and $\mathbf{R}^{-}(\mathbf{p})$, depending on the sign choice in (6):

$$
\begin{aligned}
& \xi^{+}=\frac{a+\sqrt{\left(1-a^{2}\right) \mathbf{p}^{\mathrm{T}} \mathbf{p}+1}}{\mathbf{p}^{\mathrm{T}} \mathbf{p}+1} \Rightarrow \mathbf{R}^{+}(\mathbf{p}) \\
& \xi^{-}=\frac{a-\sqrt{\left(1-a^{2}\right) \mathbf{p}^{\mathrm{T}} \mathbf{p}+1}}{\mathbf{p}^{\mathrm{T}} \mathbf{p}+1} \Rightarrow \mathbf{R}^{-}(\mathbf{p})
\end{aligned}
$$

Both matrices are orthonormal with a positive unit determinant, making them both acceptable parametrizations of a rotation. With two solutions, the mapping from GRPs to the rotation matrix is ambiguous. By using (5), the relation between the two rotations produced with (11) can be expressed as

$$
\mathbf{p}=\frac{\mathbf{q}^{+}}{q_{0}^{+}+a}=\frac{\mathbf{q}^{-}}{q_{0}^{-}+a}
$$

or, in terms of rotation axis and angle,

$$
\frac{\mathbf{n}^{+} \cos \left(\phi^{+} / 2\right)}{\sin \left(\phi^{+} / 2\right)+a}=\frac{\mathbf{n}^{-} \cos \left(\phi^{-} / 2\right)}{\sin \left(\phi^{-} / 2\right)+a}
$$

The two matrices $\mathbf{R}^{+}(\mathbf{p})$ and $\mathbf{R}^{-}(\mathbf{p})$ parametrize two very different orientations, albeit a relation between them can be established.

\subsection{Inverse mapping: rotation matrices to GRPs}

The elements of a GRP can be extracted from a rotation matrix as (see Appendix A.2)

$$
\mathbf{p}=\frac{1}{4 \underline{\xi}(a-\underline{\xi})}\left(\begin{array}{l}
r_{32}-r_{23} \\
r_{13}-r_{31} \\
r_{21}-r_{12}
\end{array}\right)
$$

with $r_{i j}$ the $i j$-th element of matrix $\mathbf{R}$ and

$$
\underline{\xi}=a \pm \frac{1}{2} \sqrt{\frac{\left(r_{32}-r_{23}\right)\left(r_{13}-r_{31}\right)}{r_{12}+r_{21}}}
$$

Note that scalar $\underline{\xi}$ should be equal to $\xi$ in (6): the underline indicates that this value is extracted from the elements of the rotation matrix, and not from the components of the GRPs. Expression (15) is also ambiguous: the inverse transformation from rotation matrix to GRPs produces two solutions. 


\subsection{Shadow set of GRPs}

The duality of the quaternion representation, $\mathbf{R}\left(q_{0}, \mathbf{q}\right)=\mathbf{R}\left(-q_{0},-\mathbf{q}\right)$, enables defining a mirroring set of GRPs, named the shadow set:

$$
\mathbf{p}_{s}=\frac{\mathbf{q}}{q_{0}-a}
$$

The quaternion components are recovered from the shadow set of GRPs as

$$
\begin{aligned}
& q_{0}=\frac{a \mathbf{p}_{s}^{\mathrm{T}} \mathbf{p}_{s} \pm \sqrt{\left(1-a^{2}\right) \mathbf{p}_{s}^{\mathrm{T}} \mathbf{p}_{s}+1}}{\mathbf{p}_{s}^{\mathrm{T}} \mathbf{p}_{s}+1}=\xi_{s}+a \\
& \mathbf{q}=\xi_{s} \mathbf{p}_{s}
\end{aligned}
$$

with

$$
\xi_{s}=\frac{-a \pm \sqrt{\left(1-a^{2}\right) \mathbf{p}_{s}^{\mathrm{T}} \mathbf{p}_{s}+1}}{\mathbf{p}_{s}^{\mathrm{T}} \mathbf{p}_{s}+1}
$$

The rotation matrix is then parameterized as

$$
\mathbf{R}\left(\mathbf{p}_{s}\right)=\mathbf{I}_{3}-2 \xi_{s}^{2}\left(\frac{a+\xi_{s}}{\xi_{s}} \boldsymbol{\Omega}_{\mathbf{p}_{s}}-\boldsymbol{\Omega}_{\mathbf{p}_{s}}^{2}\right)
$$

while the inverse mapping is

$$
\mathbf{p}_{s}=\frac{1}{4 \underline{\xi}_{s}\left(a+\underline{\xi}_{s}\right)}\left(\begin{array}{l}
r_{23}-r_{32} \\
r_{31}-r_{13} \\
r_{12}-r_{21}
\end{array}\right)
$$

with

$$
\underline{\xi}_{s}=-a \pm \frac{1}{2} \sqrt{\frac{\left(r_{23}-r_{32}\right)\left(r_{31}-r_{13}\right)}{r_{12}+r_{21}}}
$$

The following sections show the conditions under which the direct $(7,19)$ and inverse $(14,20)$ mappings determine the correct rotation matrix for a unique body rotation. The unambiguous direct mapping constitutes external consistency (i.e. the mapping produces the same rotation matrix as other parametrizations for the same body orientation) and is necessary for closing the following loops

$$
\begin{aligned}
& (\mathbf{n}, \phi) \rightarrow \mathbf{p} \rightarrow \mathbf{R}(\mathbf{p})=\mathbf{R}(\mathbf{n}, \phi) \\
& (\mathbf{n}, \phi) \rightarrow \mathbf{p}_{s} \rightarrow \mathbf{R}\left(\mathbf{p}_{s}\right)=\mathbf{R}(\mathbf{n}, \phi)
\end{aligned}
$$

The unambiguous inverse mapping constitutes internal consistency (i.e. the one-to-one mapping between a set of orientation parameters and the corresponding rotation matrix) and is necessary for closing the following loops

$$
\begin{gathered}
\mathbf{p} \rightarrow \mathbf{R}(\mathbf{p}) \rightarrow \mathbf{p}^{\prime}=\mathbf{p} \\
\mathbf{p}_{s} \rightarrow \mathbf{R}\left(\mathbf{p}_{s}\right) \rightarrow \mathbf{p}_{s}^{\prime}=\mathbf{p}_{s}
\end{gathered}
$$




\section{Internal consistency}

3.1 Internal consistency for the direct set

To ensure internal consistency for the direct set, we must close the first loop in (23). By substituting in (14) the elements of the rotation matrix expressed as function of the GRPs vector $\mathbf{p}$ in (7), the following identity is obtained:

$$
\mathbf{p}=\frac{\xi(a-\xi)}{\underline{\xi}(a-\underline{\xi})} \mathbf{p}
$$

Therefore, the fraction multiplier on the right-hand side must equal one. Using (7) and (15), the scalar $\underline{\xi}$ equals to

$$
\underline{\xi}=a \pm|a-\xi|
$$

From the definition of $\xi$ in (6), the fraction $\frac{\xi(a-\xi)}{\underline{\xi}(a-\underline{\xi})}$ is expressed as (see derivation in Appendix A.3)

$$
f=\frac{(a \pm \beta) \cdot \operatorname{sign}\left(a \mathbf{p}^{\mathrm{T}} \mathbf{p} \mp \beta\right)}{\mp a\left(1+\mathbf{p}^{\mathrm{T}} \mathbf{p}\right)-\left|a \mathbf{p}^{\mathrm{T}} \mathbf{p} \mp \beta\right|}
$$

where $\beta=\sqrt{\left(1-a^{2}\right) \mathbf{p}^{\mathrm{T}} \mathbf{p}+1}$ and the boxed $\mp$ expression is used to distinguish the sign selection in the inverse mapping (15) from the sign selection in the direct mapping (6). Depending on the sign choices, four different fractions are obtained:

$$
\begin{aligned}
& f^{++}=\frac{(a+\beta) \cdot \operatorname{sign}\left(a \mathbf{p}^{\mathrm{T}} \mathbf{p}-\beta\right)}{-a\left(1+\mathbf{p}^{\mathrm{T}} \mathbf{p}\right)-\left|a \mathbf{p}^{\mathrm{T}} \mathbf{p}-\beta\right|} \\
& f^{+-}=\frac{(a+\beta) \cdot \operatorname{sign}\left(a \mathbf{p}^{\mathrm{T}} \mathbf{p}-\beta\right)}{+a\left(1+\mathbf{p}^{\mathrm{T}} \mathbf{p}\right)-\left|a \mathbf{p}^{\mathrm{T}} \mathbf{p}-\beta\right|} \\
& f^{-+}=\frac{(a-\beta) \cdot \operatorname{sign}\left(a \mathbf{p}^{\mathrm{T}} \mathbf{p}+\beta\right)}{-a\left(1+\mathbf{p}^{\mathrm{T}} \mathbf{p}\right)-\left|a \mathbf{p}^{\mathrm{T}} \mathbf{p}+\beta\right|} \\
& f^{--}=\frac{(a-\beta) \cdot \operatorname{sign}\left(a \mathbf{p}^{\mathrm{T}} \mathbf{p}+\beta\right)}{+a\left(1+\mathbf{p}^{\mathrm{T}} \mathbf{p}\right)-\left|a \mathbf{p}^{\mathrm{T}} \mathbf{p}+\beta\right|}
\end{aligned}
$$

Table 1 Internal consistency for the GRPs

\begin{tabular}{|c|c|c|}
\hline & $a<0$ & $a>0$ \\
\hline$f^{++}=1$ & $\left\{\forall \mathbf{p} \in \mathbb{R}^{3}\right\}$ & $\left\{\mathbf{p} \in \mathbb{R}^{3} \mid \mathbf{p}^{\mathrm{T}} \mathbf{p} \leq \frac{1}{a^{2}}\right\}$ \\
$f^{+-}=1$ & $\{\emptyset\}$ & $\left\{\mathbf{p} \in \mathbb{R}^{3} \mid \mathbf{p}^{\mathrm{T}} \mathbf{p} \geq \frac{1}{a^{2}}\right\}$ \\
$f^{-+}=1$ & $\left\{\mathbf{p} \in \mathbb{R}^{3} \mid \mathbf{p}^{\mathrm{T}} \mathbf{p} \geq \frac{1}{a^{2}}\right\}$ & $\{\emptyset\}$ \\
$f^{--}=1$ & $\left\{\mathbf{p} \in \mathbb{R}^{3} \mid \mathbf{p}^{\mathrm{T}} \mathbf{p} \leq \frac{1}{a^{2}}\right\}$ & $\left\{\forall \mathbf{p} \in \mathbb{R}^{3}\right\}$ \\
\hline
\end{tabular}


A summary of the conditions that guarantee internal consistency is given in Table $^{1} 1$. Note that none of the four combinations guarantee internal consistency for the whole domain of parameter $a$. Internal consistency can be achieved, for example, by always taking the plus sign in (6) and (15) if $a<0$ $\left(f^{++}=1\right)$ and the negative sign if $a>0\left(f^{--}=1\right)$. This is a first extension of the findings in [10]: ignoring all but these two cases, a simple logic based on the sign of $a$ is sufficient to guarantee internal consistency for any rotation. However, this logic does not guarantee external consistency (see section 4).

3.2 Internal consistency for the shadow set

By applying the same reasoning to the shadow set of GRPs, the second loop in (23) yields

$$
\mathbf{p}_{s}=\frac{\xi_{s}\left(a+\xi_{s}\right)}{\underline{\xi}_{s}\left(a+\underline{\xi}_{s}\right)} \mathbf{p}_{s}
$$

Again, the fraction on the right hand-side of (28) must be one to guarantee internal consistency. An expression equivalent to (26) is thus obtained:

$$
f_{s}=\frac{\left(-a \pm \beta_{s}\right) \cdot \operatorname{sign}\left(a \mathbf{p}_{s}^{\mathrm{T}} \mathbf{p}_{s} \pm \beta_{s}\right)}{\mp a\left(1+\mathbf{p}_{s}^{\mathrm{T}} \mathbf{p}_{s}\right)+\left|a \mathbf{p}_{s}^{\mathrm{T}} \mathbf{p}_{s} \pm \beta_{s}\right|}
$$

with $\beta_{s}=\sqrt{\left(1-a^{2}\right) \mathbf{p}_{s}^{\mathrm{T}} \mathbf{p}_{s}+1}$ and the boxed $\mp$ expression is used to distinguish the sign selection in the inverse mapping (21) from the sign selection in the direct mapping (18). The four different choices are

$$
\begin{aligned}
f_{s}^{++} & =\frac{\left(-a+\beta_{s}\right) \cdot \operatorname{sign}\left(a \mathbf{p}_{s}^{\mathrm{T}} \mathbf{p}_{s}+\beta_{s}\right)}{-a\left(1+\mathbf{p}_{s}^{\mathrm{T}} \mathbf{p}_{s}\right)+\left|a \mathbf{p}_{s}^{\mathrm{T}} \mathbf{p}_{s}+\beta_{s}\right|} \\
f_{s}^{+-} & =\frac{\left(-a+\beta_{s}\right) \cdot \operatorname{sign}\left(a \mathbf{p}_{s}^{\mathrm{T}} \mathbf{p}_{s}+\beta_{s}\right)}{+a\left(1+\mathbf{p}_{s}^{\mathrm{T}} \mathbf{p}_{s}\right)+\left|a \mathbf{p}_{s}^{\mathrm{T}} \mathbf{p}_{s}+\beta_{s}\right|} \\
f_{s}^{-+} & =\frac{\left(-a-\beta_{s}\right) \cdot \operatorname{sign}\left(a \mathbf{p}_{s}^{\mathrm{T}} \mathbf{p}_{s}-\beta_{s}\right)}{-a\left(1+\mathbf{p}_{s}^{\mathrm{T}} \mathbf{p}_{s}\right)+\left|a \mathbf{p}_{s}^{\mathrm{T}} \mathbf{p}_{s}-\beta_{s}\right|} \\
f_{s}^{--} & =\frac{\left(-a-\beta_{s}\right) \cdot \operatorname{sign}\left(a \mathbf{p}_{s}^{\mathrm{T}} \mathbf{p}_{s}-\beta_{s}\right)}{+a\left(1+\mathbf{p}_{s}^{\mathrm{T}} \mathbf{p}_{s}\right)+\left|a \mathbf{p}_{s}^{\mathrm{T}} \mathbf{p}_{s}-\beta_{s}\right|}
\end{aligned}
$$

1 The case $\mathbf{p}^{\mathrm{T}} \mathbf{p}=\frac{1}{a^{2}}$ is discussed in Appendix A.4

Table 2 Internal consistency for the shadow set of GRPs

\begin{tabular}{|c|c|c|}
\hline & $a<0$ & $a>0$ \\
\hline$f_{s}^{++}=1$ & $\left\{\mathbf{p}_{s} \in \mathbb{R}^{3} \mid \mathbf{p}_{s}^{\mathrm{T}} \mathbf{p}_{s} \leq \frac{1}{a^{2}}\right\}$ & $\left\{\forall \mathbf{p}_{s} \in \mathbb{R}^{3}\right\}$ \\
$f_{s}^{+-}=1$ & $\left\{\mathbf{p}_{s} \in \mathbb{R}^{3} \mid \mathbf{p}_{s}^{\mathrm{T}} \mathbf{p}_{s} \geq \frac{1}{a^{2}}\right\}$ & $\{\emptyset\}$ \\
$f_{s}^{-+}=1$ & $\{\emptyset\}$ & $\left\{\mathbf{p}_{s} \in \mathbb{R}^{3} \mid \mathbf{p}_{s}^{\mathrm{T}} \mathbf{p}_{s} \geq \frac{1}{a^{2}}\right\}$ \\
$f_{s}^{--}=1$ & $\left\{\forall \mathbf{p}_{s} \in \mathbb{R}^{3}\right\}$ & $\left\{\mathbf{p}_{s} \in \mathbb{R}^{3} \mid \mathbf{p}_{s}^{\mathrm{T}} \mathbf{p}_{s} \leq \frac{1}{a^{2}}\right\}$ \\
\hline
\end{tabular}


Table $^{2} 2$ gives the conditions that guarantee internal consistency when using the shadow set of GRPs. The comparison of tables 1 and 2 highlights the complementary behavior of the shadow set with respect to the direct set.

\section{External consistency}

4.1 External consistency for the direct set

In order to guarantee that the correct rotation matrix is selected when choosing the sign in (6), the first identity in (5) has to be satisfied:

$$
\frac{\xi-a}{q_{0}}=1
$$

When this criterion is fulfilled, the GRPs parametrize the same rotation described by the quaternion. Using (6), the ratio can be expressed as (see derivation in Appendix A.5)

$$
g=\frac{1}{q_{0}} \frac{a\left(q_{0}^{2}-1\right) \pm\left|q_{0}+a\right|\left(1+a q_{0}\right)}{a^{2}+2 a q_{0}+1}
$$

which produces two different expressions depending on sign selection:

$$
\begin{aligned}
& g^{+}=\frac{1}{q_{0}} \frac{a\left(q_{0}^{2}-1\right)+\left|q_{0}+a\right|\left(1+a q_{0}\right)}{a^{2}+2 a q_{0}+1} \\
& g^{-}=\frac{1}{q_{0}} \frac{a\left(q_{0}^{2}-1\right)-\left|q_{0}+a\right|\left(1+a q_{0}\right)}{a^{2}+2 a q_{0}+1}
\end{aligned}
$$

The sign choice that satisfies (31) depends on the actual values of $a$ and $q_{0}$. Table 3 summarizes the conditions that guarantee external consistency. For a given $a$, neither of the two sign choices guarantees external consistency for an arbitrary rotation (through $q_{0}$ ). Also, there is no way to identify the correct sign without a priori information of the actual orientation (again via $q_{0}$ ). Moreover, a comparison of Tables 1 and 3 shows a break down of the simple decision logic discussed in Section 3. The ruling for internal consistency - a

2 The case $\mathbf{p}_{s}^{\mathrm{T}} \mathbf{p}_{s}=\frac{1}{a^{2}}$ is discussed in Appendix A.4

Table 3 External consistency for the GRPs

\begin{tabular}{|c|c|c|}
\hline & $a<0$ & $a>0$ \\
\hline$g^{+}$ & $q_{0}>0,|a|<\left|q_{0}\right|$ & $\left\{\begin{array}{l}q_{0}>0 \\
q_{0}<0,|a|>\left|q_{0}\right|\end{array}\right.$ \\
$g^{-}$ & $\left\{\begin{array}{l}q_{0}<0 \\
q_{0}>0,|a|>\left|q_{0}\right|\end{array}\right.$ & $q_{0}<0,|a|<\left|q_{0}\right|$ \\
\hline
\end{tabular}


Table 4 External consistency for the shadow set of GRPs

\begin{tabular}{|c|c|c|}
\hline & $a<0$ & $a>0$ \\
\hline$g_{s}^{+}$ & $\begin{array}{l}q_{0}>0 \\
q_{0}<0,|a|>\left|q_{0}\right|\end{array}$ & $q_{0}>0,|a|<\left|q_{0}\right|$ \\
\hline$g_{s}^{-}$ & $q_{0}<0,|a|<\left|q_{0}\right|$ & $\begin{array}{l}q_{0}<0 \\
q_{0}>0,|a|>\left|q_{0}\right|\end{array}$ \\
\hline
\end{tabular}

plus sign for (6) and (15) if $a<0$ and two negative signs if $a>0$ - would violate external consistency for some rotations (specifically in the intervals $\left\{a<0, q_{0}>0,|a|>\left|q_{0}\right|\right\}$ and $\left\{a>0, q_{0}<0,|a|>\left|q_{0}\right|\right\}$, respectively).

\subsection{External consistency for the shadow set}

By repeating the same reasoning with the shadow set of GRPs, the identity to satisfy follows from (17)

$$
\frac{\xi_{s}+a}{q_{0}}=1
$$

from which, using (18), one obtains

$$
g_{s}=\frac{1}{q_{0}} \frac{a\left(1-q_{0}^{2}\right) \pm\left|q_{0}-a\right|\left(1-a q_{0}\right)}{a^{2}-2 a q_{0}+1}
$$

The expression above splits, depending on the sign choice, into

$$
\begin{aligned}
& g_{s}^{+}=\frac{1}{q_{0}} \frac{a\left(1-q_{0}^{2}\right)+\left|q_{0}-a\right|\left(1-a q_{0}\right)}{a^{2}-2 a q_{0}+1} \\
& g_{s}^{-}=\frac{1}{q_{0}} \frac{a\left(1-q_{0}^{2}\right)-\left|q_{0}-a\right|\left(1-a q_{0}\right)}{a^{2}-2 a q_{0}+1}
\end{aligned}
$$

Table 4 summarizes the conditions that guarantee external consistency for the shadow set of GRPs as function of the values of $a$ and $q_{0}$. The same conclusions for the direct set apply to the shadow set: external consistency cannot be globally guaranteed by either sign choice, and additional information on the actual orientation is needed. However, Tables 3-4 also show the complementary behavior of the direct and shadow sets. As shown in the next section, the direct and shadow sets can be used jointly to enforce both internal and external consistency.

\section{Overall consistency}

Whereas internal consistency can always be enforced by a simple sign decision logic - triggered by the values of parameter $a$, see Tables 1-2 - one further 
Table 5 Overall consistency for the direct and shadow set of GRPs

\begin{tabular}{|c|c|c|}
\hline & $a<0$ & $a>0$ \\
\hline $\mathbf{p}^{\mathrm{T}} \mathbf{p}<\mathbf{p}_{s}^{\mathrm{T}} \mathbf{p}_{s}$ & $\left\{\begin{array}{l}f^{--} \\
g^{-}\end{array}\right.$ & $\left\{\begin{array}{l}f^{++} \\
g^{+}\end{array}\right.$ \\
$\mathbf{p}_{s}^{\mathrm{T}} \mathbf{p}_{s}<\mathbf{p}^{\mathrm{T}} \mathbf{p}$ & $\left\{\begin{array}{l}f_{s}^{++} \\
g_{s}^{+}\end{array}\right.$ & $\left\{\begin{array}{l}f_{s}^{--} \\
g_{s}^{-}\end{array}\right.$ \\
\hline
\end{tabular}

step is needed to guarantee external consistency without the need of acquiring additional information of the actual orientation.

Let us consider the following ratio:

$$
r=\frac{\mathbf{p}^{\mathrm{T}} \mathbf{p}}{\mathbf{p}_{s}^{\mathrm{T}} \mathbf{p}_{s}}=\frac{\left(q_{0}-a\right)^{2}}{\left(q_{0}+a\right)^{2}}
$$

The scalar $r$ is larger than one if $a$ and $q_{0}$ have opposite signs $\left(q_{0} a<0\right)$, and smaller than one if $a$ and $q_{0}$ are either both positive or both negative $\left(q_{0} a>0\right)$. It follows that, by working with the direct set $\mathbf{p}$ if $r<1$, and with the shadow set $\mathbf{p}_{s}$ if $r>1$, the following inequalities are verified:

$$
\left\{\begin{array}{l}
\mathbf{p}^{\mathrm{T}} \mathbf{p} \leq \frac{1}{\left(q_{0}+a\right)^{2}} \leq \frac{1}{a^{2}}, q_{0} a>0 \quad \text { for } \quad r<1 \\
\mathbf{p}_{s}^{\mathrm{T}} \mathbf{p}_{s} \leq \frac{1}{\left(q_{0}-a\right)^{2}} \leq \frac{1}{a^{2}}, q_{0} a<0 \quad \text { for } \quad r>1
\end{array}\right.
$$

By comparing Tables 1 and 3 (or Tables 2 and 4 for the shadow set), it it easy to verify that the inequalities in (38) imply that both internal and external consistencies are achieved by a combined use of the direct and shadow GRPs, with the simple logic of working with the set of smaller norm and using the sign decision logic given in Table 5. This result is a generalization of the combined use of direct and shadow parameters for $a= \pm 1$ proposed in [6], [7] and [8].

\section{Conclusions}

This work complements the literature on the use of generalized Rodrigues parameters, also known as symmetric stereographic orientation parameters, for representing rigid body orientations. It is shown how to resolve the ambiguities inherent to both the direct (parameters to rotation matrix) and the inverse (rotation matrix to parameters) transformations by a combined use of the direct and shadow set of parameters. The decision logic summarized in Table 5 enables using the GRPs for representing rotations without incurring in singularities. Note that this does not violate any of the two theorems on the topological impossibility of having a one-to-one global rotation parameterization without singular points with less than five parameters [11]-[12]: the combined use of two sets of GRPs makes the parameterization analyzed in this work a six-element representation. 


\section{Acknowledgment}

The author would like to thank Andreas Brack (German Research Center for Geosciences - GFZ) and Bethany Kroese (US Air Force Research Laboratory) for their valuable comments on the original manuscript.

This is a pre-print of an article published in the Journal of the Astronautical Sciences. The final authenticated version is available online at:

https://doi.org/10.1007/s40295-019-00183-2.

\section{Conflict of interest}

The author states that there is no conflict of interest.

\section{A Appendix}

A.1 Proof: $\mathbf{R}(\mathbf{p}) \in \mathbb{S O}(3), \forall \mathbf{p} \in \mathbb{R}^{3}$

The product $\mathbf{R}^{\mathrm{T}}(\mathbf{p}) \mathbf{R}(\mathbf{p})$ reads, using (7)

$$
\mathbf{R}^{\mathrm{T}}(\mathbf{p}) \mathbf{R}(\mathbf{p})=\mathbf{I}_{3}+4 \xi^{2}\left(\mathbf{I}_{3}+\xi^{2} \boldsymbol{\Omega}_{\mathbf{p}}^{2}-(a-\xi)^{2} \mathbf{I}_{3}\right) \boldsymbol{\Omega}_{\mathbf{p}}^{2}
$$

By substituting $\Omega_{\mathbf{p}}^{2}=\mathbf{p} \mathbf{p}^{\mathrm{T}}-\mathbf{p}^{\mathrm{T}} \mathbf{p} \mathbf{I}_{3}$, one obtains

$$
\mathbf{R}^{\mathrm{T}}(\mathbf{p}) \mathbf{R}(\mathbf{p})=\mathbf{I}_{3}
$$

since $\Omega_{\mathrm{p}} \mathbf{p}=0$.

The determinant of matrix $\mathbf{R}(\mathbf{p})$ can be derived as follows:

$$
\operatorname{det}\left(\mathbf{R}^{\mathrm{T}}(\mathbf{p}) \mathbf{R}(\mathbf{p})\right)=(\operatorname{det} \mathbf{R}(\mathbf{p}))^{2}=1
$$

Thus, $\operatorname{det} \mathbf{R}(\mathbf{p})= \pm 1$, but since the determinant for $\mathbf{p}=\mathbf{0}$ is +1 , and $\mathbf{R}(\mathbf{p})$ is a continuous function of the GRPs, the determinant is identically one for any real-valued vector $\mathbf{p}$. This demonstrates that expression (7) always produces a proper rotation matrix.

\section{A.2 Inverse map}

The orientation matrix in terms of GRPs is explicitly given as

$$
\begin{gathered}
\mathbf{R}(\mathbf{p})=\mathbf{I}_{3}+2 \xi^{2}\left[\frac{a-\xi}{\xi} \boldsymbol{\Omega}_{\mathbf{p}}+\boldsymbol{\Omega}_{\mathbf{p}}^{2}\right] \\
=\left[\begin{array}{ccc}
1-2 \xi^{2}\left(p_{2}^{2}+p_{3}^{2}\right) & 2 \xi^{2} p_{1} p_{2}+2 \xi(a-\xi) p_{3} & 2 \xi^{2} p_{1} p_{3}-2 \xi(a-\xi) p_{2} \\
2 \xi^{2} p_{1} p_{2}-2 \xi(a-\xi) p_{3} & 1-2 \xi^{2}\left(p_{1}^{2}+p_{3}^{2}\right) & 2 \xi^{2} p_{2} p_{3}+2 \xi(a-\xi) p_{1} \\
2 \xi^{2} p_{1} p_{3}+2 \xi(a-\xi) p_{2} & 2 \xi^{2} p_{2} p_{3}-2 \xi(a-\xi) p_{1} & 1-2 \xi^{2}\left(p_{1}^{2}+p_{2}^{2}\right)
\end{array}\right]
\end{gathered}
$$

from which relations (14)-(15) are easily obtained.

Note that, should the sum $r_{12}+r_{21}$ in (15) approach zero, it is sufficient to use one of the two alternatives

$$
\begin{aligned}
\underline{\xi} & =a \pm \frac{1}{2} \sqrt{\frac{\left(r_{32}-r_{23}\right)\left(r_{21}-r_{12}\right)}{r_{13}+r_{31}}} \\
& =a \pm \frac{1}{2} \sqrt{\frac{\left(r_{13}-r_{31}\right)\left(r_{21}-r_{12}\right)}{r_{23}+r_{32}}}
\end{aligned}
$$


The same reasoning applies to the shadow set of GRPs.

Expressions (14)-(20) are specifically worked out to analyze internal consistency properties. From a computational perspective, it is easier to use the quaternion inversion formulas to obtain the inverse mapping:

$$
q_{0}=\frac{1}{2} \sqrt{1+\operatorname{tr}(\mathbf{R})} \quad ; \quad \mathbf{q}=\frac{1}{4 q_{0}}\left(\begin{array}{l}
r_{23}-r_{32} \\
r_{31}-r_{13} \\
r_{12}-r_{21}
\end{array}\right)
$$

from which

$$
\mathbf{p}=\frac{1}{\sqrt{1+\operatorname{tr}(\mathbf{R})}(2 a+\sqrt{1+\operatorname{tr}(\mathbf{R})})}\left(\begin{array}{l}
r_{23}-r_{32} \\
r_{31}-r_{13} \\
r_{12}-r_{21}
\end{array}\right)
$$

This function diverges in two cases. The first is for rotations about any axis and angle $\phi=(2 k+1) \pi(k \in \mathbb{Z})$, for which $\operatorname{tr}(\mathbf{R})=-1$, corresponding to a null quaternion scalar component. Obviously, this is not a degenerate point, and the issue is circumvented by taking one of the following alternative inverse functions [13]:

$$
\begin{aligned}
& \gamma=\frac{1}{2} \sqrt{1+r_{11}-r_{22}-r_{33}} \\
& \mathbf{p}=\frac{1}{4 \gamma a+r_{23}-r_{32}}\left(\begin{array}{c}
(2 \gamma)^{2} \\
r_{12}+r_{21} \\
r_{13}+r_{31}
\end{array}\right)
\end{aligned}
$$

$$
\begin{aligned}
& \gamma=\frac{1}{2} \sqrt{1+r_{11}-r_{22}-r_{33}} \\
& \mathbf{p}=\frac{1}{4 \gamma a+r_{23}-r_{32}}\left(\begin{array}{c}
(2 \gamma)^{2} \\
r_{12}+r_{21} \\
r_{13}+r_{31}
\end{array}\right)
\end{aligned}
$$

$$
\begin{aligned}
& \gamma=\frac{1}{2} \sqrt{1+r_{11}-r_{22}-r_{33}} \\
& \mathbf{p}=\frac{1}{4 \gamma a+r_{23}-r_{32}}\left(\begin{array}{c}
(2 \gamma)^{2} \\
r_{12}+r_{21} \\
r_{13}+r_{31}
\end{array}\right)
\end{aligned}
$$

Since both $\left(q_{0}, \mathbf{q}\right)$ and $\left(-q_{0},-\mathbf{q}\right)$ are valid parameterizations of the same attitude matrix $\mathbf{R}$, for each of the four possible inversions (45)-(48) both the direct and the shadow GRPs can be recovered by simply inverting the sign of scalar $a$. It is then straightforward to apply the logic reported in Table 5 to select the appropriate set that guarantees both internal and external consistency.

The second degenerate point is at a rotation for which $2 a+\sqrt{1+\operatorname{tr}(\mathbf{R})}=0$, corresponding to a quaternion scalar component $q_{0}=-a$, which is indeed a singularity point for the GRPs direct representation. This singularity is avoided by switching to the shadow set through inversion of the sign of parameter $a$.

\section{A.3 Derivation of identity $(26)$}

Using identity (25) in (24), one obtains

$$
\frac{\xi(a-\xi)}{\underline{\xi}(a-\underline{\xi})}=\frac{\xi(a-\xi)}{\mp|a-\xi|(a| \pm| a-\xi \mid)}
$$


Using (6), expression (49) works out as

$$
\begin{aligned}
\frac{\xi(a-\xi)}{\underline{\xi}(a-\underline{\xi})} & =\frac{(a \pm \beta)\left(a \mathbf{p}^{\mathrm{T}} \mathbf{p} \mp \beta\right)}{\mp\left|a \mathbf{p}^{\mathrm{T}} \mathbf{p} \mp \beta\right|\left(a\left(1+\mathbf{p}^{\mathrm{T}} \mathbf{p}\right) \pm\left|a \mathbf{p}^{\mathrm{T}} \mathbf{p} \mp \beta\right|\right)} \\
& =\frac{a \pm \beta}{\mp a\left(1+\mathbf{p}^{\mathrm{T}} \mathbf{p}\right)-\left|a \mathbf{p}^{\mathrm{T}} \mathbf{p} \mp \beta\right|} \frac{a \mathbf{p}^{\mathrm{T}} \mathbf{p} \mp \beta}{\left|a \mathbf{p}^{\mathrm{T}} \mathbf{p} \mp \beta\right|}
\end{aligned}
$$

with $\beta=\sqrt{\left(1-a^{2}\right) \mathbf{p}^{\mathrm{T}} \mathbf{p}+1}$. The last ratio on the right-hand side in (50) only retains the sign of the scalar $a \mathbf{p}^{\mathrm{T}} \mathbf{p} \mp \beta$, and identity (26) is thus obtained.

\section{A.4 Rotations characterized by $\mathbf{p}^{\mathrm{T}} \mathbf{p}=\mathbf{p}_{s}^{\mathrm{T}} \mathbf{p}_{s}=\frac{1}{a^{2}}$}

Functions (26) and (29) may take an indeterminate value when the squared norm of the GRPs approaches the value $\frac{1}{a^{2}}$. However, any of the alternative inversion functions (46)-(48) can be used to study internal consistency. Such rotations are characterized by symmetric rotation matrices (the scalar component of quaternion is null, thus $\xi=a$ and $\xi_{s}=-a$ ), and $\mathbf{R}(\mathbf{p})=\mathbf{R}(-\mathbf{p})=\mathbf{R}\left(\mathbf{p}_{s}\right)$, thus the direct and the shadow GRPs can be swapped without altering the result.

\section{A.5 Derivation of identity (32)}

Substituting (6) into (31) gives

$$
\frac{\xi-a}{q_{0}}=\frac{1}{q_{0}}\left(\frac{a \pm \sqrt{\left(1-a^{2}\right) \mathbf{p}^{\mathrm{T}} \mathbf{p}+1}}{1+\mathbf{p}^{\mathrm{T}} \mathbf{p}}-a\right)
$$

From relation (4) one obtains $\mathbf{p}^{\mathrm{T}} \mathbf{p}=\frac{1-q_{0}^{2}}{\left(q_{0}+a\right)^{2}}$. Expression (51) then works out as

$$
\begin{aligned}
\frac{\xi-a}{q_{0}} & =\frac{1}{q_{0}} \frac{ \pm \sqrt{\left(q_{0}+a\right)^{2}\left(a q_{0}+1\right)^{2}}-a\left(1-q_{0}^{2}\right)}{a^{2}+2 a q_{0}+1} \\
& =\frac{1}{q_{0}} \frac{ \pm\left|q_{0}+a\right|\left|a q_{0}+1\right|+a\left(q_{0}^{2}-1\right)}{a^{2}+2 a q_{0}+1}
\end{aligned}
$$

Since both $a$ and $q_{0}$ are only defined in the interval $[-1 ;+1]$, the term $\left|a q_{0}+1\right|$ is always larger than, or equal to, zero. Expression (32) is then obtained.

\section{References}

1. J. Stillwell, Naive Lie Theory. Springer, 2008.

2. A. J. Kirillov Jr., An introduction to Lie groups and Lie algebras. Cambridge University Press, Cambridge, 2008.

3. W. R. Hamilton, "On Quaternions; or on a new System of Imaginaries in Algebra," The London, Edinburgh and Dublin Philosophical Magazine and Journal of Science (3rd Series), vol. xxv-xxxvi, pp. Edited by David R. Wilkins, 2000, 1844-1850.

4. O. Rodrigues, "Des lois géométriques qui régissent les déplacements d'un système solide dans l'espace, et la variation des coordonnées provenant de ses déplacements considérés indépendamment des causes qui peuvent les produire," Journal de Mathématiques Pures et Appliquées, vol. 5, pp. 380-440, 1840. 
5. J. W. Gibbs, The collected works of J. Willard Gibbs, vol.2. Yale University Press, New Haven, CT, USA, 1948.

6. T. F. Wiener, "Theoretical Analysis of Gimballess Inertial Reference Equipment Using Delta-modulated Instruments," PhD dissertation, Massachusetts Institute of Technology, Cambridge, MA, US, 1962.

7. S. R. Marandi and V. J. Modi, "A Preferred Coordinate System and the Associated Orientation Representation in Attitude Dynamics," Acta Astronautica, vol. 15, no. 11, pp. 833-843, 1987.

8. P. Tsiotras, "New Control Laws for the Attitude Stabilization of Rigid Bodies," IFAC Proceedings Volumes, vol. 27, no. 13, pp. 321-326, 1994.

9. H. Schaub and J. L. Junkins, "Stereographic Orientation Parameters for Attitude Dynamics: A Generalization of the Rodrigues Parameters," Journal of the Astronautical Sciences, vol. 44, no. 1, pp. 1-19, 1996.

10. C. M. Southward, J. R. Ellis, and H. Schaub, "Symmetric stereographic orientation parameters applied to constrained spacecraft attitude control," The Journal of the Astronautical Sciences, vol. 55, no. 3, pp. 389-405, 2007.

11. H. Hopf, "Systeme Symmetrischer Bilinearformen und Euklidische Modelle der Projektiven Räume," Vierteljahrschr. Naturforsch. ges. Zürich, vol. 85, pp. 165-177, 1940.

12. J. Stuelpnagel, "On the Parameterization of the Three-Dimensional Rotation Group," SIAM Review, vol. 6, no. 4, pp. 422-430, 1964.

13. A. R. Klumpp, "Singularity-free extraction of a quaternion from a direction-cosine matrix," Journal of Spacecraft and Rockets, vol. 13, no. 12, pp. 754-755, 1976. 